\title{
Clustering Schemes for D2D Communications Under Partial/No Network Coverage
}

\author{
Lu Qianxi ${ }^{1}$, Miao Qingyu ${ }^{1}$, Gabor Fodor $^{2}$, Nadia Brahmi $^{3}$ \\ Ericsson Research \\ ${ }^{1}$ Beijing, China; ${ }^{2}$ Stockholm, Sweden; ${ }^{3}$ Aachen, Germany \\ email: \{qianxi.lu, qingyu.miao, gabor.fodor, nadia.brahmi $\} @$ ericsson.com
}

\begin{abstract}
Device-to-Device (D2D) communication has been proposed to deal with the significant amount of the local traffic by enabling devices to directly connect to each other when being in proximity. In order to start direct communications, a device must first discover that other devices are in the proximity and that they are relevant for a certain context (i.e. can offer relaying to the network). This task becomes particularly challenging in out-of-network coverage. In this paper, we propose three clustering schemes that assist a device discovering and associating with other devices considered relevant in a given context. We compare the efficiency of the proposed approaches in terms of discovery ratio, clustering time and energy consumption and study the tradeoff between these performance metrics that need to be considered when selecting one of the grouping schemes. In particular, the simulation results prove that grouping devices based on their capabilities taking into account the exchanged radio signal strengths ensure, in most of the scenarios, the balance between high discovery ratio, short latency and energy efficiency. ${ }^{1}$
\end{abstract}

Keywords-D2D; Device Discovery; Clustering

\section{INTRODUCTION}

Device-to-Device (D2D) communication has been identified as a promising technology component for future wireless systems to meet the increasing traffic demands and to handle the large number of connected devices expected for the next decades. By allowing devices to autonomously discover other nearby devices and to establish direct connections without user intervention, D2D technology will enable a wide range of local-area services (e.g. proximity-based commercial services, location-based social applications) [1, 2]. An increasing attention was particularly drawn to using D2D networks as an underlay to the cellular networks where the short-range links share the radio resources with the assistance of the network $[3,4]$. In addition to enhancing the spectrum efficiency, this improves the coverage of the network while maintaining low power consumption.

Recently, the usage of D2D technology was also considered for the National Security and Public Safety (NSPS) support scenarios [5, 6]. The 3rd Generation Partnership Project (3GPP) is currently investigating the delivery of such services

\footnotetext{
${ }^{1}$ This work has been partially performed in the framework of the FP7 project ITC 317669 METIS.
}

over the LTE network. Even though, according to many studies, network-assisted D2D is likely to provide highperformance, more functionalities are still needed in case of out-of-network coverage to guarantee the services requirements especially in terms of latency and energy efficiency $[7,8]$. The challenge is to develop a D2D framework that takes full advantage of the infrastructure whenever available and ensures the coordination of local communications between devices in areas where the infrastructure was partially or completely destroyed.

One fundamental component of the D2D framework is the devices (peer) discovery that enables neighboring devices to autonomously discover each other. This question has been addressed over the past years mainly with proposals on fully distributed device discovery schemes [9]. Examples include systems based on Wi-Fi technology such as Wi-Fi direct and Nokia Instant Community (NIC) running on unlicensed without infrastructure support [10, 11]. However, device discovery in such systems costs much time and energy especially when a large range is required. Another D2D system is the Flashlink recently proposed in [12], which defines a greedy resource allocation scheme to distribute the discovery resources among devices in a decentralized way. The network assistance is only needed for synchronization.

In general, the key technique used in these schemes is to define specially designed beacon signals that devices can broadcast and capture, so that nearby devices can detect the proximity as well as some characteristics of the broadcasting devices. In order for discovery to work, the beacon capturing device must be able to decode the information encoded in the beacon signal. In other words, the beacon signal must reach a certain signal-to-noise-and-interference (SINR) threshold at the capturing device. Nevertheless, when the number of devices exceeds the number of available peer discovery resources (PDRs), two nodes may use the same resource leading to collisions and thus preventing the capturing devices from detecting both devices. The greedy scheme proposed by Flashlink copes with such situations and creates improvement in the system performances compared to the random resource allocation. However, studies indicate that these graph coloring algorithms can not go beyond a certain discovery rate within a limited discovery time in cases the density of devices becomes very high and every device is broadcasting its discovery beacon [13]. 
Most of the existing solutions proposed so far consider situations in which each device is interested in discovering all the devices in its surrounding. However, it may happen that a device is only interested in discovering and establishing direct connections with only few devices considered relevant in a given context. For instance, in the NSPS scenarios it is more relevant that a device discovers the devices being carried by the members of the rescue team or the devices having special capabilities to forward messages to the network and reach the emergency center (fire brigades, police, etc, ) in order to speed up the emergency intervention.

Therefore, the purpose of this paper is to propose a clusterbased discovery scheme where clusters are formed in a way that allows devices to discover the relevant devices in their surrounding and build D2D connections in out-of-network coverage. The basic idea of the clustering is to let so called cluster head capable devices to take over some of the functionalities of a cellular base station when the cellular infrastructure becomes damaged or dysfunctional. In practice such cluster head devices can be implemented by ad-hoc (vehicle carried) or handheld devices by special capabilities in terms of transmit power, UL/DL spectrum usage, security capabilities and other functionalities.

The reminder of the paper is structured as follows. The next section presents the system model and the assumptions made for our study. Next, section III describes the D2D clustering concept, including the devices discovery, cluster-head selection and different alternatives used for devices grouping. Section IV discusses simulation results and evaluates the gain of different approaches in terms of discovery-rate, latency and energy consumption. Finally, section V states conclusions and points out future works.

\section{SYSTEM MODEL}

In this section, we introduce the network model and the assumptions considered for the rest of the paper.

\section{A. Assumptions}

In our study, we consider the cases where only a subset of the devices or none of them is under the cellular coverage. The availability of the network coverage in combination with the capabilities of the devices (e.g. the maximum transmission power, power level, etc.) is used to distinguish between two types of devices:

- Type-1UEs: represent the user terminals (UE) and devices capable of acting as cluster-heads $(\mathrm{CH})$ and able to control afterwards the formation of the D2D links and to manage the resources usage among a group of D2D devices associated with it. For each of these devices a pre-computed metric is defined as a function combining the capabilities of the devices $(\mathrm{CH}$ capable or not, maximum power allowed), the remaining power level and the availability or not of the network coverage. The reason for including the network availability is to assign higher priority for the devices under the network coverage to form clusters and take advantage of the network assistance for managing the D2D peers. This also means that each device has to upgrade its own capability when it gets under (or out of) network coverage and triggers a new clustering procedure.

- Type-2 UEs: such devices can only act as cluster members (slaves) and therefore they are always controlled by appropriate type-1UEs that they select as cluster head to assist them for the establishment of the D2D links and during the communication phase. In this paper we assume that two Type-2 UEs out of the coverage of a cellular base station or a $\mathrm{CH}$ cannot communicate directly with one another, which corresponds to the situation of today's legacy UEs.

We assume that only type-1UEs are allowed to broadcast their beacon signals for discovery. Based on exchanged signals, some of these devices will designate themselves as clusterheads, the rest of type-1 UEs and the type-2 UEs will each join a cluster-head and hence forming a cluster structure. More details on the clustering procedure will be described in next section.

\section{B. Discovery Model}

Concerning the resources used for the discovery, we assume that one beacon signal is sent using one Peer Discovery Resource (PDR) defined in the form of OFDM Physical Resources Blocks (PRB) in, for example, LTE-A frame structure. We also assume that only certain frames are used for discovery (e.g. one frame every 10 LTE-A frames) and within each discovery frame we assume a predefined number of PDRs. The structure of the PDRs is out of the scope of this paper.

In this context, whether a device is discovered or not by another neighboring device is defined by the quality of received SINR measured from the beacon signal. Therefore, we use the SINR threshold as the criterion to evaluate the discovery independently of potential collisions that might happen when different devices use the same resource to broadcast their beacon signals. In fact, as long as the SINR measured at the receiving device (Rx_UE) from a certain transmitter (Tx_UE) exceeds the threshold, we consider this latter to be discovered by Rx_UE. The value of the SINR threshold could be pre-defined depending on the decoding techniques used. Thanks to recent advances even very low SINR (e.g. below $0 \mathrm{~dB}$ ) could be decoded and thus increasing the discovery coverage around the device.

Additionally, it is assumed that the devices can decode different beacon signals in different frequency simultaneously.

\section{DEVICE CLUSTERING ALTERNATIVES}

The clustering procedure consists of three main phase: the first step is to identify the nodes with the best capabilities to play the role of cluster heads. The second step is to select the appropriate cluster-head among all the potential ones. Finally, each device will connect to the corresponding $\mathrm{CH}$ to build a cluster structure. 


\section{A. Cluster-heads Identification}

During this phase, each type-1 UE continuously broadcasts its beacons including its identifier and its pre-defined metric using one PDR selected among the set of resources preconfigured for the discovery purpose. However, without any coordination, it may happen that two sending devices meet in space, time and frequency leading to collisions which reduces the discovery rate and prolongs the discovery procedure. Such scenarios become even more frequent when the number of PDRs is insufficient compared with the number of active type1 UEs and where some resources have to be reused. To address this problem and minimize the risk of collisions, we define a transmission probability Ptx according to which a device decides in each time slot if it should broadcast its beacon or remains in listening mode. In addition, we introduce a randomization procedure to reduce even further the probability of having the collisions repetitively between the same devices and allow the use of resources more efficiently. According to this scheme, in every time slot in which a device decides to send, it picks randomly one single PDR from the multiple resources available in the frequency dimension.

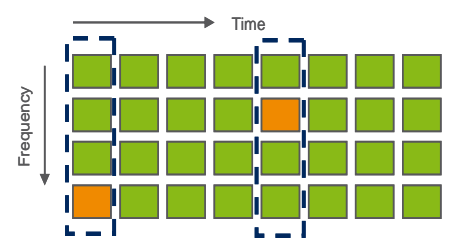

Fig. 1. For each time slot (one unit in the time dimension), each master decides to transmit or to listen with probability ptr. Then it picks a random PDR from the multiple PDRs in the frequency dimension.

In parallel, every device, when receiving and successfully decoding a beacon, stores the identifier of the sender and the corresponding metric.

The main goal at this phase is to build local awareness among type- 1 devices that will allow them delegating the controlling functions only to a few devices in order to make the D2D resource management simpler and more efficient. At the end of the first step, we consider that each type-1 UE has at least received one beacon signal from each of its neighboring type-1UEs and has exploited that to build the knowledge about its local surrounding. If no beacon signal is received during this phase the device will assume itself being out of coverage of any other type-1 UE and identify itself as a cluster-head.

Next, using the information collected during this phase, each type-1 UE compares its own metric with the metrics of its neighbors. The devices having the highest metrics will designate themselves as cluster-heads (CHs). Other type-1 UEs having lower metrics will be identified as slaves (which we will also refer to as non-CH UE) and they have to join one of the cluster-heads.

\section{B. Phase 2: UE Clustering}

In this phase, each non- $\mathrm{CH}$ device has to select the appropriate $\mathrm{CH}$ and associate with it. This association is done based on the $\mathrm{CH}$ indication that each type-1 device after taking the new role of $\mathrm{CH}$ has to include it in its beacons in addition to its metric as well as the signals received from all potential
CHs. At this stage, three grouping schemes could be used: cluster-head driven, hybrid and threshold-based approaches (as shown in Figure 2).

1) Cluster-head Driven: One simple way for grouping the devices with their appropriate cluster-heads is that only the $\mathrm{CHs}$ continue broadcasting their beacons including their metrics. The rest of types-1 UEs and type-2 UEs use the received metrics to associate with the $\mathrm{CH}$ having the highest metric value among all the cluster-head candidates exceeding a certain threshold (e.g. SINR above 5-10dB). This threshold value could be pre-configured in order to ensure a good link quality between any slave-master peer. This approach has the advantage of reducing the number of active devices competing for the same resources, which decreases the collision risk and improves the SINR quality received within a given range. This is expected to make the discovery process more efficient not only in terms of discovery rate and time but also in terms of energy consumption. Nevertheless, according to this alternative once a device is identified as a $\mathrm{CH}$, it is not allowed to be a slave of another $\mathrm{CH}$ which prevents inter-cluster communications even in case devices from two different clusters are close to one another. Furthermore, since the type-1 UEs have no information about the density and the distribution of the type-2 devices, it may happen that a type-1 UE identifies itself as a slave (i.e. lower metric than its neighbors) although it could serve as a cluster-head for a group of devices being outof coverage of any of the existing CHs (i.e. very low SINR received from the existing $\mathrm{CHs}$ ).

2) Hybrid: In this clustering alternative, every type-1UE, independently whether it is a $\mathrm{CH}$ or a slave, continues broadcasting its beacon using the same random transmission pattern as was used during the first phase until all the remaining UEs find their cluster-heads or the maximum discovery rate is achieved. As opposed to the $\mathrm{CH}$ driven clustering scheme, this approach allows the possibility of building new clusters using the nodes initially designated as slaves which increases the probability of a device being under the coverage of one cluster. In particular, one device is allowed to act as a $\mathrm{CH}$ in one cluster and be a slave in a second cluster, thus facilitating the relaying of information between different clusters whenever the need rises. However, the drawback of such scheme is the high beacon load it generates in the system which increases the collision risks and thereby the time and the energy needed for $\mathrm{CH}$ discovery.

3) Threshold-based: Our third clustering alternative takes in account the quality of signals received from different clusterheads to estimate if a type-1 UE is out-of-cluster coverage and so if it should keep broadcasting its beacons. More precisely, if a type-1 UE is already identified as slave but the maximum received signal from any of the $\mathrm{CHs}$ is below a certain threshold (e.g., SNR = 20/40/60dB), the device is said to be located at a cluster edge and so it has to continue sending out beacons in order to provide coverage for other nodes in the surrounding. Since the slaves are allowed to participate in $\mathrm{CH}$ selection based on the signal quality, this increases the 
probability of having every device under the coverage of at least one $\mathrm{CH}$ while maintaining a reduced overlapping area between clusters. One advantage of such a scheme is ensuring low inter-cluster interferences by controlling the cluster range which may at a later stage allow the support of multi-hop relaying.

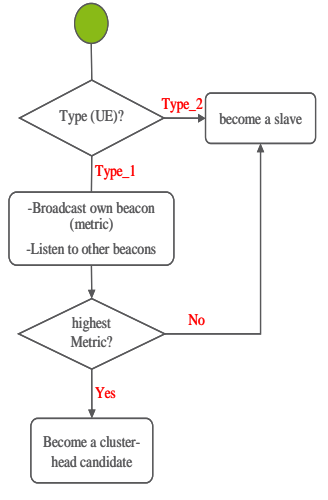

(a) Cluster-head identification

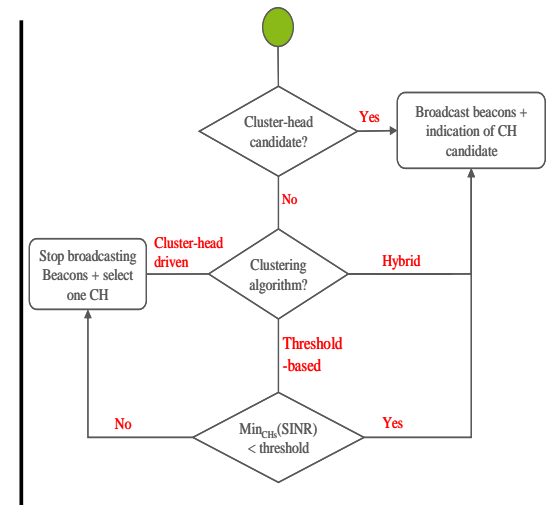

(b) Type-1 UE clustering
Fig. 2. Flow charts for step 1 and step 2: based on their types and the clustering approach used, the type-1 UEs decide to broadcast or not their beacons.

\section{Phase 3: Connection Establishment}

Once each node has identified the appropriate cluster-head, the next step is to establish an association with this $\mathrm{CH}$. For this purpose, the slave can, for example, send a request to connect to the candidate $\mathrm{CH}$; if this request is accepted then the slave will have a control plane connection to the $\mathrm{CH}$ which will be used by the latter to provide synchronization and to schedule the resource allocation for different D2D pairs. This association could also be done without any additional signaling exchange and where the slaves only follow the synchronization and the RRM information broadcasted by the $\mathrm{CH}$. The synchronization procedure and the RRM algorithms are out of the scope of this paper.

Figure 3 shows an example of the cluster structures built using the three proposed clustering schemes for a given devices distribution.

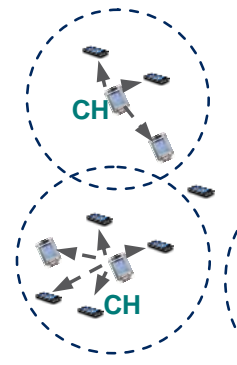

(a)

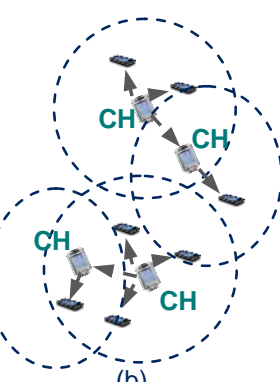

(b)

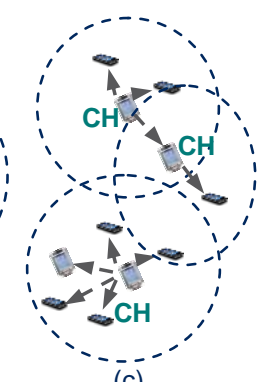

(c)
Fig. 3. (a) Cluster-head Driven, (b) hybrid and (c) threshold-based clustering approaches

In this cluster-head driven approach, less clusters are formed which results in some UEs remaining in out-of coverage. The hybrid scheme allows all type-1 UEs to broadcast their beacons and thus more clusters are formed. Finally, in the threshold-based clustering the clusters are formed based on the signal strengths received from other cluster-heads and so additional clusters (compared to those created in the first alternative) are only created if UEs remains which allows to cover isolated devices.

The rest of this paper is devoted to the evaluation of different clustering approaches. As it will be illustrated in the next section, each alternative has its benefits and drawbacks. The goal here is to find the balance between cluster coverage, the latency required for structuring the D2D network into clusters and the energy consumption needed for the device grouping.

\section{PERFORMANCES ANALYSIS}

In this section we consider an area equivalent to one cell in a cellular system with inter-site distance ISD $=500 \mathrm{~m}$, in which equal loads of type-1 UEs and type-2 UEs are uniformly dropped. We assume that all devices will operate in D2D mode and that the path loss between devices can be modeled using 3GPP Home eNB model [13] with 1 building per sector and $35 \%$ of the users indoor.

To gain insight into the tradeoff between the performance measures of interest for the D2D clustering procedure, we simulate the performance of the proposed alternatives for different density and traffic load: we consider UE loads ranging from 20 to 500 UEs per cell and beacon transmission probabilities from 0.1 to 0.5 .

\section{A. Evaluation Metrics}

In order to compare different proposed clustering approaches, we define the following performance metrics.

1) Convergence Ratio: This metric measures the efficiency of the clustering algorithm by calculating the ratio of devices that are able to discover the right cluster-head within a certain time duration (e.g. 25s). Using the knowledge about the metrics, path losses between devices and the discovery SINR threshold we are able to identify which slave should connect to which $\mathrm{CH}$ : the devices that connect to the right $\mathrm{CH}$ are identified as converged devices as opposed to the un-converged ones that remain isolated either due to a high beacon collision rate or low signals received from all the $\mathrm{CHs}$ pre-selected during the first phase.

2) Convergence Time: For the converged UEs, this metric further looks at the time spent before these devices select their corresponding CHs.

3) Energy Consumption: The type-1 UEs consume energy when they transmit their own beacons or receive signals from other type-1 UEs, whereas the type-2 UEs only consume energy when receiving and decoding beacons. Correspondingly, the energy consumption is computed seperately for type-1 UEs and type-2 UEs. However, assuming that a transmission consumes more power than a reception, we only consider, in this study, the number of time intervals when the UE is active for beacons transmission [11]. 


\section{B. Cluster Coverage}

We define the coverage of a cluster as the area where the beacon signals of the $\mathrm{CH}$ reach all the devices in the area with an SINR value above the discovery threshold so that they can correctly be decoded. An estimation of the number of UEs being at the coverage of at least one $\mathrm{CH}$ shows that only using the cluster-head driven approach, some devices remain out of coverage while the other two alternatives provide full coverage by allowing a higher number of type-1 UEs to send their beacons. Nevertheless, as shown in figure 4, the number of UEs out-of coverage decreases significantly when the tolal density of devices increases (which implies higher umber of CHs).

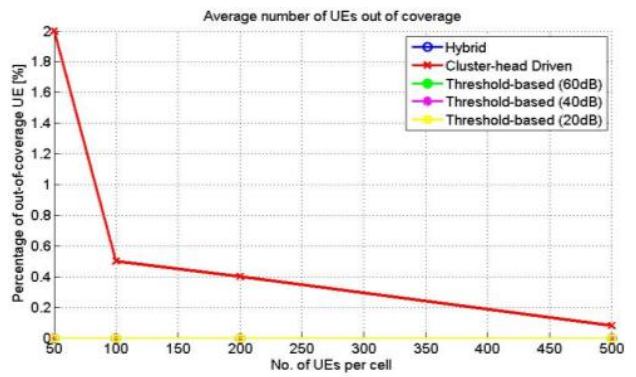

Fig. 4. Number of UEs out of cluster-coverage: in cluster-driven approach since only few nodes are desginated as $\mathrm{CHs}$ without any consideration of the signal strengths received from these $\mathrm{CHs}$, some UEs remain out of coverage in particular if the density of type-1 UEs becomes low to cover the whole network.

\section{Convergence Ratio Vs Convergence Time}
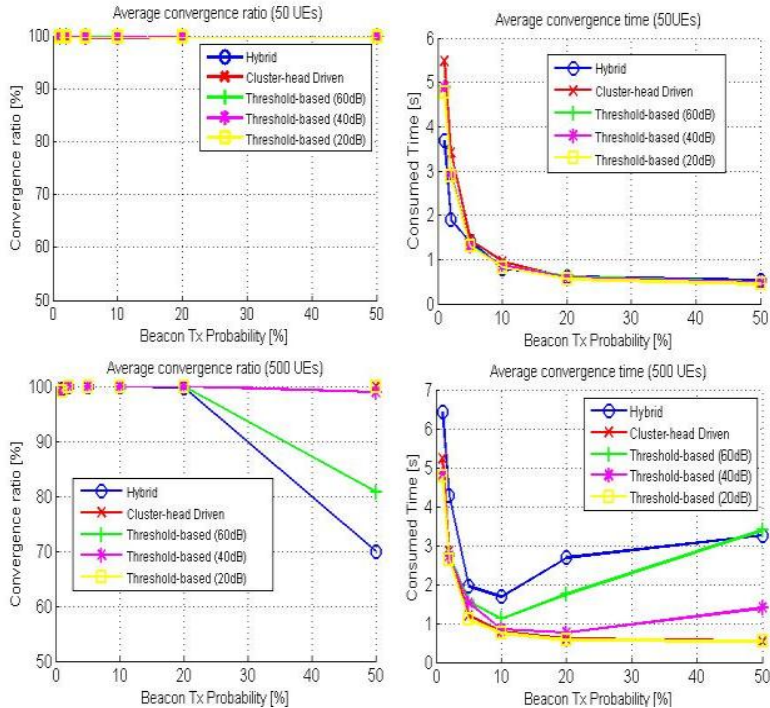

Fig. 5. Convergence ratio and convergence latency achieved by different clustering approaches with different system loads. When the beacon load is high, the collision risk becomes more important and reduces the discovery rate and increases the time required to form clusters between neigboring D2D candidates.

As we can see in the figure above, when the density of devices is low, the three clustering approaches provide similar convergence ratio within the same latency. The reason for that is that the resources available for discovery are higher than the number of devices which makes the probability of having two devices sending within the same PDR very low and thus maximizing the discovery rate of the type-1 UEs. However, when the density of devices competing for the same resources increases this leads to high collision risk, in particular using the hybrid clustering scheme where all the type-1 UEs are transmitting their beacons, hence leading to lower and slower convergence. The same behavior is observed in the third alternative when the transmission threshold is set very high (60dB, meaning that all the type-1 UEs that receive a signal to noise ratio less then $60 \mathrm{~dB}$ have to continue transmitting their beacons). More precisely, the higher the threshold becomes the lower the coverage of one cluster is considered which introduces higher number of active devices in the system and leads to higher beacon loads and collisions risk.

\section{Energy Consumption}
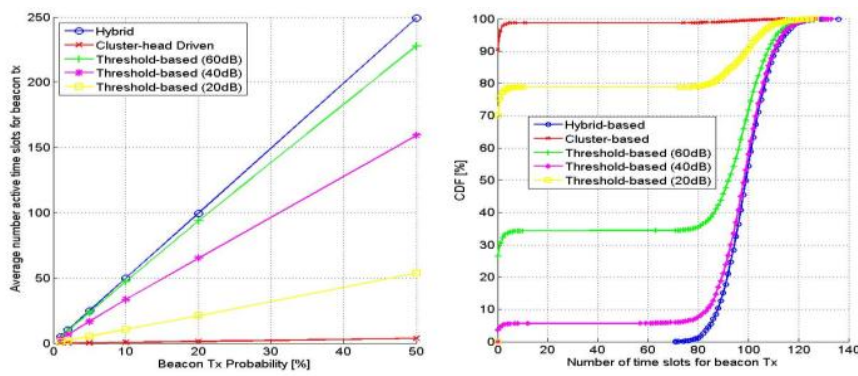

Fig. 6. Energy required for discovery and clustering is lower when only few nodes $(\mathrm{CHs})$ are allowed to transmit their beacon signals.

As mentioned previously, the energy consumption is proportional to the number of the active slots for the beacon transmissions. The results in Figure 5 show that the clusterhead-driven approach is very energy efficient compared to the hybrid approach where every potential $\mathrm{CH}$ is broadcasting its beacons in particular when a high transmission probability is chosen. Nevertheless, this energy efficiency comes to the detriment of the coverage as illustrated in the previous sections. On the other hand, a low value selected for the transmission threshold in the third alternative ensures a balance between the energy consumption and the convergence ratio.

\section{E. Multi-layer Clusters and Inter-cluster Interference}

In multi-layer cluster, one cluster-head could be a slave of a second cluster. We define the highest level in the hierarchy as a root-cluster. Figure 6 compares the number of layer in the clusters created by each clustering approaches.

As expected, the cluster-head driven approach does not allow inter-cluster communications and so the total number of clusters is similar to the number of root clusters. In the other two alternatives, the multi-layer clustering increases the total number of clusters by enabling up to 3 layers in a cluster. This will introduce more inter-cluster interferences which require coordination. However, if we integrate inter- $\mathrm{CH}$ coordination within each root cluster, such approaches may provide better performances with less interference to manage between independent clusters (root-clusters). This becomes particularly efficient if we ensure small overlapping areas between these independent clusters. 

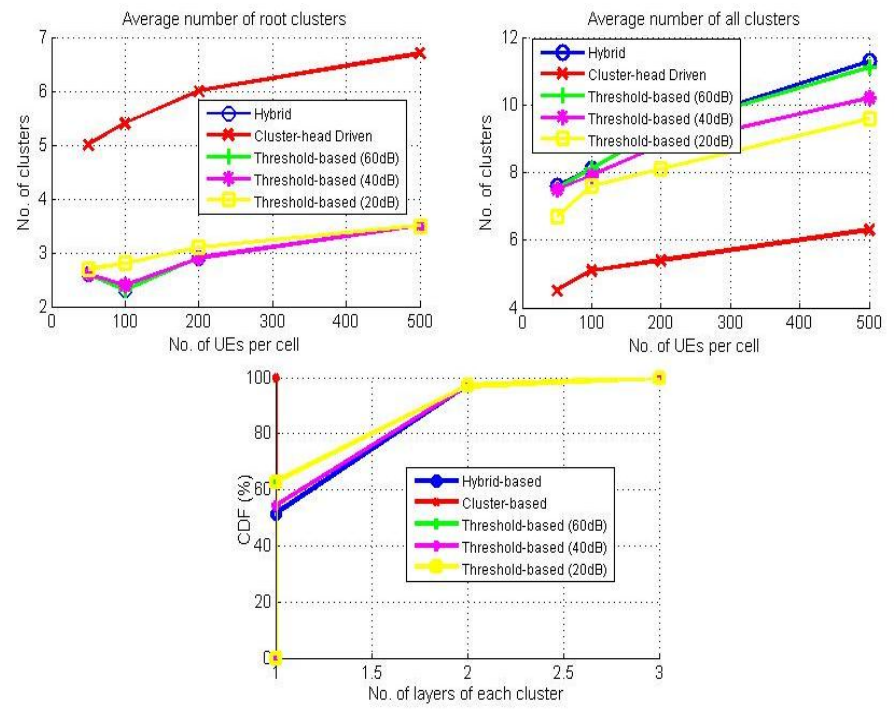

Fig. 7. The number of clusters and their structures: in the cluster-head driven scheme only root clusters are created as opposed to the hybrid and the threshold-based approaches where multi-layer clustering reduces the number of root clusters by creating hierarchy inside the root clusters.

It should also be observed that selecting a low beacon transmission threshold (20dB) allows inter-cluster communications as well but with less total number of clusters and it generates less inter-cluster interferences. This observation is confirmed when measuring the number of devices suffering from inter-cluster interferences as indicated in Figure 7. The threshold-based clustering seems to be the best approach to achieve the balance between the cluster coverage and the inter-cluster interferences. The overlapping areas between clusters should be large enough to provide coverage for all the devices but not too small to generate high interference between clusters.
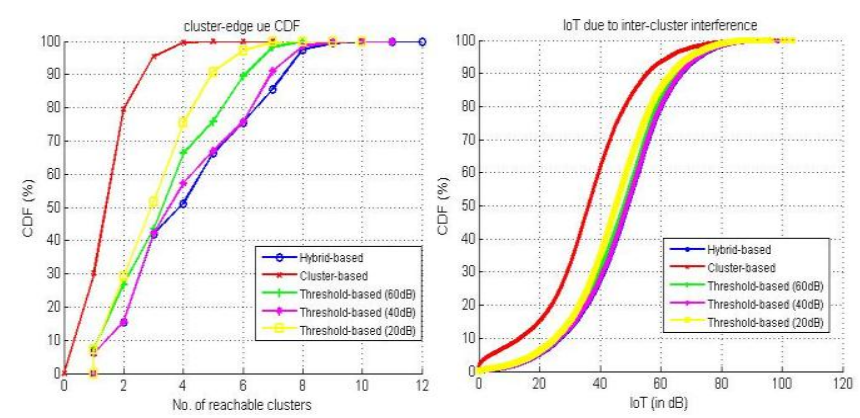

Fig. 8. Inter-cluster intereference: in cluster-driven approach no inter-cluster communication is allowed therefore less devices are affected by inter-cluster intereference, whereas more devices experience intereference in the two other two other alternatives due to larger overlapping areas between clusters.

\section{CONCLUSIONS}

In this paper we developed clustering schemes for device discovery and D2D communication in out-of-network coverage scenarios. In particular, the grouping procedures allow a device to discover and connect to a cluster head device capable of taking over some of the functionalities of cellular base stations in emergency or disaster situations. The proposed approaches were implemented in a system simulator and tested for different users' densities and traffic load. The results indicate the benefit of selecting a few nodes for beacon signals instead of all-to-all broadcast in terms of discovery ratio, latency and energy required for building the D2D network. In addition, by enabling multi-layer clustering, the third scheme extends the range of one cluster-head and facilitates the coordination between clusters. This will be particularly useful for radio resource management during the communication phase in addition to allowing multi-hop communication when needed.

\section{REFERENCES}

[1] M. Corson, R. Laroia, J. Li, V. Park, T. Richardson, G. Tsirtsis, "Toward proximity-aware internetworking", IEEE Wireless Communications 17(6), 26-33 (2010)

[2] Kaasinen, E., "User Needs for Location-Aware Mobile Services", Personal and Ubiquitous Computing, Volume 7, Issue 1, May 2003.

[3] K. Doppler, M. Rinne, C. Wijting, C. B. Ribeiro, K. Hugl, "Device-todevice communication as an underlay to LTE-advanced networks," IEEE Communications Magazine, vol. 47, no. 12, pp. 42-49, Dec. 2009.

[4] Gabor Fodor, et al., Design Aspects of Network AssistedDevice-toDevice Communications, IEEE Communications Magazine,March 2012.

[5] 3GPP website announcement "FCC selects LTE for USA Public Safety" http://www.3gpp.org/FCC-selects-LTE-for-USA-Public

[6] 3GPP website link to FCC announcement of selection of LTE for USA public safety "FCC TAKES ACTION TO ADVANCE NATIONWIDE BROADBAND COMMUNICATIONS FOR AMERICA'S FIRST RESPONDERS" http://www.3gpp.org/IMG/pdf/psltedoc-304244a1.pdf

[7] M. Belleschi, G. Fodor and A. Abrardo, "Performance Analysis of a Distributed Resource Allocation Sceheme for D2D Communications", IEEE Workshop on Machine-to-Machine Communications, Houston, TX, USA, December 2011

[8] C.-H. Yu et al, "Power Optimization of Device-to-Device Communication Underlaying Cellular Communication," IEEE Int'l. Conf. Commun., ICC, Dresden, Germany, June 2009.

[9] S.Vasudevan, D.Towsley and D.Goeckel. "Neighbor Discovery in Wireless Networks and the Coupon Collector's Problem", ACM Mobicom '09, Beijing, China, September 2009.

[10] http://www.wi-fi.org/discover-and-learn/wi-fi-direct

[11] K.Doppler, C.Ribeiro and J.Kneckt. "Advances in D2D Communications: Energy Efficient Service and Device Discovery Radio", International Conference on Wireless Communication, Vehicular Technology, Information Theory and Aerospace and Electronic Systems Technology, March 2011

[12] F.Baccelli, N.Khude, R.Laroia, J.Li, T.Richardson, S.Shakkottai, S.Tavildar and X.Wu. "On the Design of Device-to-Device Autonomous Discovery", 4th International Conference on Communication Systems and Networks, Bangalore, India, January 2012.

[13] 3GPP TR 36.814: 3rd Generation Partnership Project; Technical Specification Group Radio Access Network; Further Advancements for E-UTRA Physical Layer Aspects

[14] Zhe Li, "Performance Analysis of Network Assisted Neighbor Discovery Algorithms. (Master Thesis Report). Royal Institute of Technology, XR-EE-RT 2012:026, Stockholm, 2012. http://urn.kb.se/resolve?urn=urn:nbn:se:kth:diva-117696 